\title{
Invasive Placentation - Comparison between Ultrasound - Colour Doppler and Magnetic Resonance Imaging
}

\author{
Naresh Kumar. $\mathbf{M}^{1}$, Sreedevi.T' ${ }^{2}$ K. Vigneshraja ${ }^{3}$, N. Anil Kumar ${ }^{4}$ \\ ${ }^{1}$ Senior Resident, Department of Radiodiagnosis, GMC, Secunderabad, ${ }^{2}$ Associate Professor, Department of Radiodiagnosis, \\ GMC, Secunderabad, ${ }^{3}$ Junior resident, Department of Radiodiagnosis, GMC, Secunderabad, ${ }^{4}$ Associate Professor, Department \\ of Radiodiagnosis, GMC, Secunderabad, India
}

Corresponding author: Dr. Sreedevi T., Associate Professor, Department of Radiodiagnosis, GMC, Secunderabad, India

DOI: http://dx.doi.org/10.21276/ijcmsr.2019.4.4.41

How to cite this article: Naresh Kumar.M, Sreedevi.T, K. Vigneshraja, N. Anil Kumar. Invasive placentation comparison between ultrasound-colour doppler and magnetic resonance imaging. International Journal of Contemporary Medicine Surgery and Radiology. 2019;4(4):D172-D176.

\section{A B S T R A C T}

Introduction: Histopathological diagnosis of invasive placenta is possible only after delivery. Accurate prenatal identification of placental invasion allows for optimaltiming, site of delivery and maternal counseling regarding future fertility. Study aimed to compare the sensitivity and specificity of ultrasound with colour doppler and MRI in diagnosing placental invasion. Material and methods: 40 pregnant women with clinically diagnosed placenta previa with high risk of invasive placentation based on clinical history were analyzed. Abnormal invasiveness was assessed using established Doppler sonography criteria, further evaluation by MRI using standardized criteria for detection and characterization of placental invasiveness was done. All the cases were correlated with surgical findings.

Results: MRI is more sensitive and specific than USG-Colour Doppler in the detection of invasive placenta.

Conclusion: USG-Doppler and MRI complement each other in diagnosing Invasive placenta. However, MRI is superior to USG in invasive placenta particularly in evaluating placenta percreta and invasion in cases of posteriorly located placenta previa.

Keywords: Placenta, Invasion, Accreta, Increta, Percreta

\section{INTRODUCTION}

Histopathological diagnosis of invasive placenta is possible only after delivery. Accurate prenatal identification of placental invasion allows for optimal timing and site of delivery, maternal counseling regarding future fertility. Noninvasive techniques that do not use ionizing radiation such as ultrasonography (US) and magnetic resonance (MR) imaging are the modality of choice for diagnosis of invasion. ${ }^{1}$ Sonography is the first imaging modality for evaluation of the placenta in the ante partum period. Color and power Doppler techniques permit visualization of placental vascularity i.e. vascular lakes with turbulent flow on colour flow mapping (peak systolic velocity $>15 \mathrm{~cm} / \mathrm{s}$ ). MR imaging has the advantage of high soft-tissue contrast resolution; however, it has lower spatial resolution compared with US and therefore is usually reserved as a complementary technique for equivocal findings at US and if additional information is required. Study aimed to compare the sensitivity and specificity of ultrasound with colour doppler and MRI in diagnosing placental invasion.

\section{MATERIAL AND METHODS}

It was a prospective observational study conducted at Gandhi Hospital, Secunderabad for 18 months (from Aug'17 to Feb'19) which included 40 patients with suspected invasion.
As a routine protocol for suspected invasive placenta, USG trans abdominal/trans vaginal, colour Doppler and MRI were done for these patients.

\section{Inclusion criteria}

The study included pregnant women with suspected placental invasion based on the clinical picture of per vaginal bleeding/ hematuria/pain abdomen and with past history of LSCS, $\mathrm{D} \& \mathrm{C}$ or myomectomy.

\section{Exclusion criteria}

Contraindications to MRI like metallic implants, claustrophobia etc.,

\section{Imaging}

Pelvic sonography was done with Esoate my lab class c Medical Systems and GE logic v5 expert equipments using a $3.5 \mathrm{MHz}$ curved, 7.5 $\mathrm{MHz}$ linear electronic array transducer, $8 \mathrm{MHz}$ Transvaginal transducer. Grayscale B-mode sonography was first used to assess the placental tissue localization and shape, followed by an assessment by superimposing color/power Doppler over suspected and potential regions of interest. MRI was performed on a $1.5 \mathrm{~T}$ (MagnetomAvento, Siemens Medical System, Erlangen, Germany) superconducting magnet using a 16 channel transmit-receive body coil. A small amount of fluid in the urinary bladder aided the evaluation of the 
uterine and bladder serosa; therefore, all MR examinations were obtained with a partially full bladder. Following a localizer scan, sagittal, axial and coronalT2 TRUFI (FOV - $360 \mathrm{~mm}$, TR - 4.2, TE - 2.0, Slice Thickness - $4.5 \mathrm{~mm}$, Phase Oversampling - 38\%, Distance Factor - 60, receiver bandwidth - $476 \mathrm{~Hz}$ )were acquired during breath-hold. Next axial and coronal T2 HASTE (FOV - $360 \mathrm{~mm}$, TR 1000, TE - 86, Slice thickness $-5 \mathrm{~mm}$, Phase oversampling - 0, Distance Factor - 50) were acquired during breathhold.

\section{RESULTS}

Out of 40 cases 33(82.5\%)had previous history of LSCS(one LSCS in 19, two LSCS in 13, three LSCS in 01), 02(5\%) cases had D\&C and 01(2.5\%) case had Myomectomy. 24 women had normally attached placenta which were easily removed during delivery. Of these, On USG-Colour Doppler 22 were predicted correctly (true negative) and 2 were labeled as invasive placenta (false positive) (Table 1). On MRI, 23 were predicted correctly (true negative) and 1 was labelled

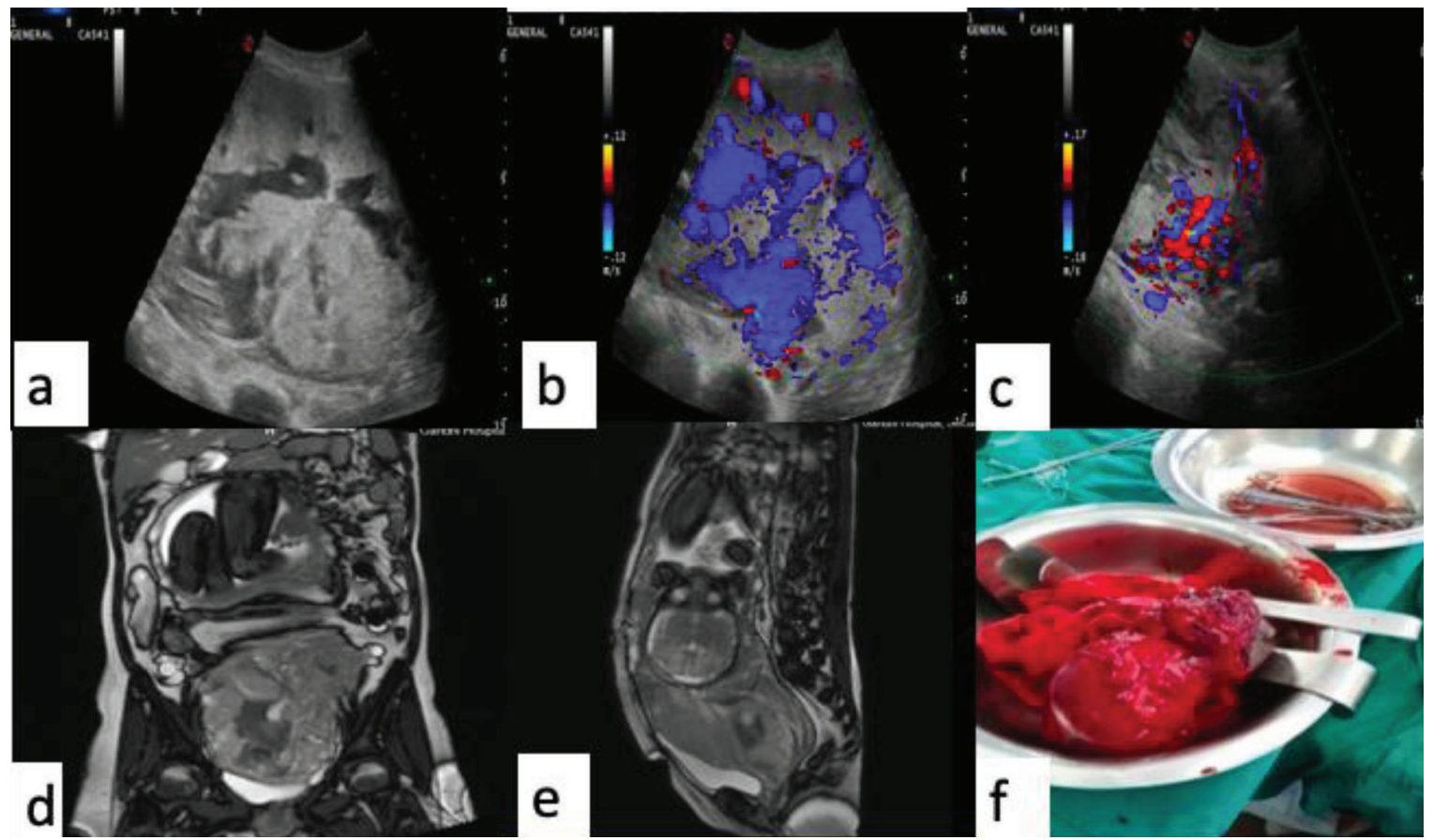

Figrue-1: a) Complete placenta previa with heterogeneous placenta and multiple intraplacentallacunae. B) Turbulent flow in intraplacentallacunae. c) thinning of myometrium with vascularity in bladder myometrial interphase. d) Trufi T2W Coronal image shows Heterogeneous complete placenta previa with multiple intraplacentallacunae. e) Trufi T2W Sagittal image shows Bulge over Urinary Bladder with loss of chemical shift artifact. f) Post Hysterectomy specimen shows Placenta percreta.

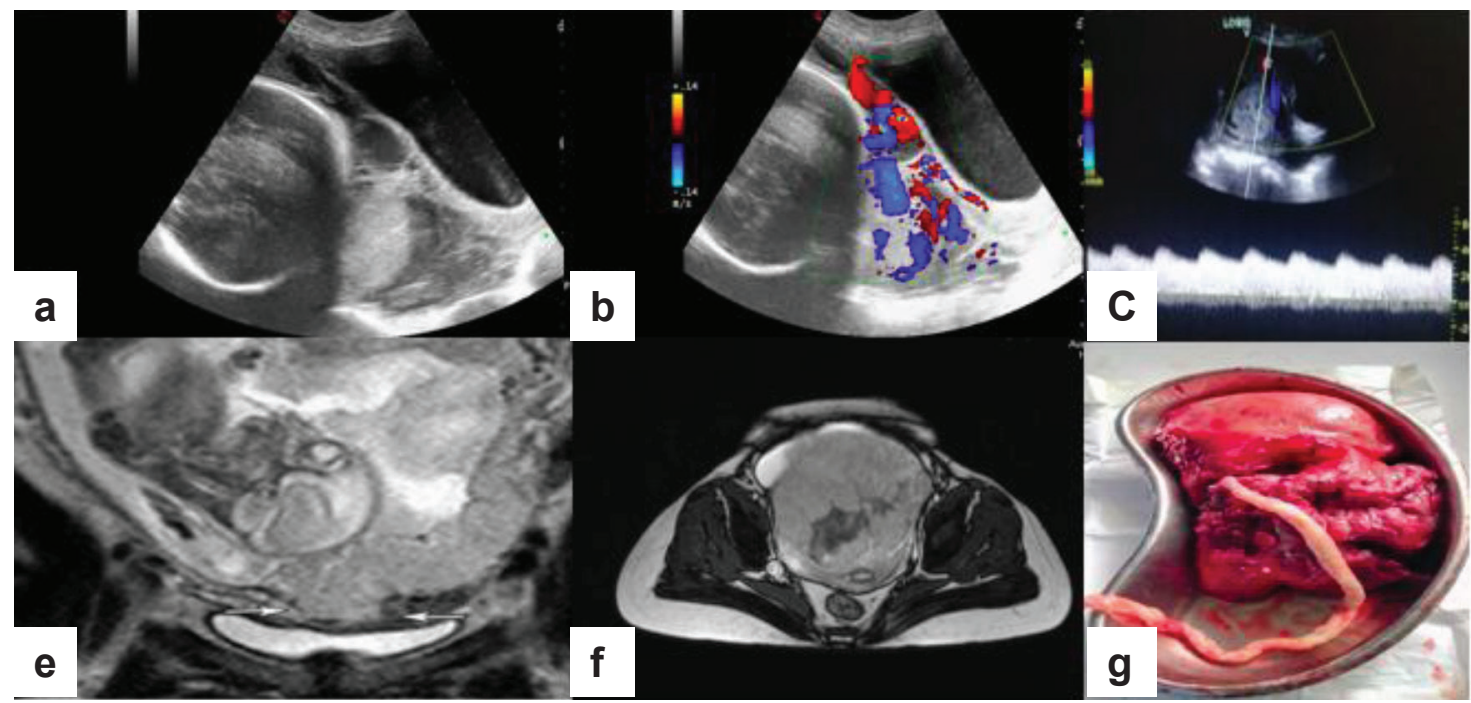

Figure-2: a) Complete placenta previa with heterogeneous placenta and multiple intraplacental lacunae within and loss of retro placental lucency; b) Colour uptake at bladder myometrial junction; c) Turbulent colour uptake and arterial spectral pattern of lacunae; d) Trufi T2W Coronal Image shows focal interruption of bladder myometrial interphase with flow voids and bulge on bladder wall; e) Trufi T2W Axial Image shows Intraplacental lacunae with few dark T2 dark bands; f) Post Op Hysterectomy specimen s/o Placenta Percreta 


\begin{tabular}{|l|c|c|c|}
\hline \multirow{2}{*}{ USG- Colour Doppler } & \multicolumn{3}{|c|}{ Intra - Operative } \\
\cline { 2 - 4 } & Positive & Negative & Total \\
\hline Positive & 14 & 2 & 16 \\
\hline Negative & 2 & 22 & 24 \\
\hline Total & 16 & 24 & 40 \\
\hline
\end{tabular}

Table-1: Comparison of USG-doppler findings with surgical findings for evaluating placental invasion

\begin{tabular}{|l|c|c|c|}
\hline \multirow{2}{*}{ MRI } & \multicolumn{3}{|c|}{ Intra - Operative } \\
\cline { 2 - 4 } & Positive & Negative & Total \\
\hline Positive & 15 & 1 & 16 \\
\hline Negative & 1 & 23 & 24 \\
\hline Total & 16 & 24 & 40 \\
\hline Table-2: Comparison of MRI with surgical findings for the \\
evaluation of invasive placenta \\
\hline
\end{tabular}

as invasive placenta (false positive). Sixteen patients had surgical confirmation of placental adhesion of which 15 were predicted correctly by MRI (true positive) and 1 was missed by MRI (False Negative) (Table 2). On USG - DOPPLER, 14 were correctly predicted as invasion (True Positive) and 2 were missed (False Negative). Sensitivity of MRI is $93 \%$ and that of USG is $87.5 \%$. Specificity of MRI is $95.8 \% \%$ and that of USG is $91.6 \%$.Thus,MRI is more sensitive and specific than USG. The positive predictive value of MRI is $93 \%$ and that of USG is $87.5 \%$. The negative predictive value for MRI is $95.8 \%$ and that for USG Doppler is $91.6 \%$.On the basis of intraoperative findings, these cases were classified into placenta accrete/ placenta increta $(n=13)$, and placenta percreta $(n=3)$. All cases with invasive placenta had low lying placenta, located in the anterior wall 14 out of 16 cases and posterior wall 2 out of 16 cases.

Among the individual sonographic and color Doppler signs mentioned for detection of invasive placenta, turbulence in visualized lacunae was the most accurate sign, with a sensitivity of $87.5 \%$ and a specificity of $91.6 \%$. However, presence of Doppler flow in these lacunae, even on applying power Doppler mode, was less accurate in detecting placental invasion and had a sensitivity of $56 \%$ with an overall diagnostic accuracy of $77 \%$.Dilated sub placental vessels with pulsatile flow and hypervascularity in the bladder-placenta interface was present in 3 cases of placenta percreta and not present in all other 13 accreta/increta cases.

Among the individual signs for identification of invasive placenta in MRI, presence of dark intraplacental bands was the most accurate sign present in 14 out of 15 cases

Markers for detecting invasion of the wall of the urinary bladder in cases of placenta percreta included loss of myometrial lining at the bladder interface on sonography, superior tenting of bladder wall, and bladder interface hypervascularity on color Doppler. Among the various MRI signs of invasive placenta, loss of chemical shift artifacts ("India-ink line") at the bladder-myometrial interface, was a sensitive and specific sign for detection of vesical wall invasion, identified in all 3 percreta cases. One case out of 16 has been detected incidentally associated with fetal anomaly of omphalocele which is terminated at $2^{\text {nd }}$ trimester by the will of mother.

\section{DISCUSSION}

Massive obstetric hemorrhage is still the leading cause of pregnancy-related deaths, and invasive placenta remains one of the major culprits of pregnancy related bleed. ${ }^{2}$ Invasive placenta is a potentially life-threatening condition, which includes various abnormalities of placental implantation in which the placenta invades the myometrium. Placenta accreta involves myometrial invasion, placenta increta involves deep myometrial invasion, and placenta percreta invades through the serosal layer of the uterus with potential invasion of adjacent bladder or bowel loops. However, we have used the term "invasive placenta" as a blanket term to describe any amount of abnormal placental invasiveness, as often done in the literature. Accurate identification of extent of invasion helps the surgeons to plan the nature of hysterectomy and thus decreasing blood loss and morbidity. Ultrasound with color Doppler imaging is presently the mainstay of diagnosis of invasive placenta. The ultrasonographic findings described in diagnosis of invasive placenta are presence of placenta previa, lacunae, reduced myometrial thickness, and loss of retro placental clear space. ${ }^{1}$ However, in our experience, loss of retro placental space is often found in normal pregnancy, and reduced myometrial thickness, also being a subjective parameter, is unreliable in diagnosing invasive placenta. Furthermore, the interface between the placenta and the myometrium is often focally indistinct, making this sign erroneous. Irregular anechoic areas within the placenta, i.e. lacunae, were the most predictive ultrasonographic sign to detect placenta accrete/increta/percreta in our study with a sensitivity of $87.5 \%$ and a positive predictive value of $87.5 \%$. These lacunae, first described by Finberg and Williams, have been the strongest ultrasonographic findings for placenta accreta. Intraplacental lacunae are indistinct anechoic structures of varying size and shape that are found in the placental parenchyma showing turbulent flow, differentiating them from placental lakes. ${ }^{3}$ Power and color Doppler have been suggested to demonstrate turbulence in placental lacunae in previous studies. Twickler et al. reported that all cases of placenta accreta had turbulent flow in placental lacunae. ${ }^{4}$ However, in our study, Doppler could demonstrate lacunar turbulence in only (12 out of 14) cases. Lacunar turbulence was more reliably detected by moving internal echogenicity on B mode US, exhibiting $92 \%$ sensitivity and $86 \%$ positive predictive value. Various studies have demonstrated that color Doppler imaging along with US has a high sensitivity (82-86\%) and specificity (92-97\%) in the diagnosis of placenta accreta. ${ }^{1,3,4}$ Besides demonstration of lacunar flow, other color Doppler features that have been described to detect placenta accreta are interface hypervascularity, with abnormal blood vessels linking the placenta to the bladder with high diastolic arterial blood flow, markedly dilated peripheral sub placental vascular channels with pulsatile venous-type flow over the uterine cervix, and lacking the peripheral sub placental hypoechoic zone. All these features were assessed in our study and were found reasonably accurate in the evaluation of placenta accreta. Bladder interface hypervascularity and dilated sub-placental vasculature were found in $18.75 \%(3 / 16)$ cases (Figure1). 
Abnormally increased vascularity at the placenta- bladder interface is assumed to represent abnormal vessels linking bladder and placenta in cases of placenta percreta. ${ }^{5}$ Interface hypervascularity has a positive predictive value of $100 \%$ in detecting placenta percreta. Hence, increased vascularity at the placenta-bladder interface might represent sub placental hypervascularity in the myometrium overlying the bladder. This was also supported by the fact that in all three patients in whom dilated sub placental vascularity was found, bladder interface hypervascularity was also noted whereas it was absent in all the other thirteen patients with only placenta accreta. Moreover, in the study by Chou etal., this sign yielded two false positive results in detecting placenta accreta owing to bladder varices due to neovascularized vessels from previous Cesarean deliveries. ${ }^{6}$

There have been many studies evaluating the role of MRI in the diagnosis of placenta accreta with reported sensitivities ranging from $38 \%$ by Lam et al. to $88 \%$ by Warshak et al. ${ }^{7,8}$ Dwyer et al. found no statistical difference in the sensitivity or specificity between US and MRI in diagnosing invasive placenta. ${ }^{9}$ MRI features that have been described to detect placenta accreta are increased placental thickness, exophytic uterine masses, abnormal uterine bulging, intraplacental hemorrhage, dark intraplacental bands, intraplacental flow voids, indistinct bladder wall margin, tenting of bladder, thinning of myometrium seen beneath placenta, marked heterogeneity of placental signal intensity, and indistinct placenta-myometrial interface. Of these parameters, placental heterogeneity, uterine bulge, dark intra-placental bands, and intra-placental hemorrhages are the most useful (Figure2).

Abnormal uterine bulging had a high sensitivity in our study with only 8 out of 15 cases having normal uterine contour. Bulging has been thought to be due to an abnormally tense myometrium created by the placental invasion or by the frequently thickened and hemorrhagic placenta in these cases. Intra-placental hemorrhages were noted in 9 out of 15 cases. 14 out of the 15 patients with invasive placenta were found to have dark intraplacental bands on T2W images which are thought to represent abnormal bands of fibrous tissue. ${ }^{10}$ The heterogeneous signal in abnormally invasive placenta could be due to the products of intra-placental hemorrhage and sonographically visible lacunae and was seen in 15 cases. It should be noted that the heterogeneity in placenta accreta is distributed throughout the placenta unlike large sub chorionic hematoma which may causes focal placental heterogeneity.

The importance of diagnosing bladder wall invasion is highlighted from one case in which the bladder could not be evaluated properly. Surgery had a fatal outcome due to torrential bleed owing to the presence of unanticipated bladder wall invasion by placenta percreta. Since this painful experience, we learned to assess the bladder wall with a full bladder. In cases of bladder wall invasion, hysterectomy is modified by using wide margins of surgical excision to avoid traversing the highly vascular placenta.

One important feature in the ultrasonographic diagnosis of placental invasion is the irregularity and hypervascularity of the uterine serosal-bladder wall complex. No surrogate
MRI feature of this sign has been described in the literature. In cases of placenta percreta with bladder invasion, loss of chemical shift artifact was noted at the bladder-uterine interface. The chemical shift artifact or India ink artifact is seen at the interface of fluid-filled organs or visceral structures with surrounding abdominal fat in TRUFI. Our study revealed that loss of chemical shift artifact at bladdermyometrial interface predicted bladder invasion in 3 out of 15 cases.

A major limitation of this study was the small sample size. Another limitation was that the interpretation of imaging features in MRI was made without blinding of the findings of US and color Doppler they were compared retrospectively and results were compared with intraoperative findings only not histopathological findings. The strength of our study was its prospective nature and thus elimination of biases which accompanies in analysis of retrospectively evaluated cohort. In conclusion, this study shows that both MRI and Doppler sonography are useful for detection of invasive placenta. However, MRI is a better predictor of bladder invasion, and invasion in case of posteriorly located placenta previa thus justifying the use of this expensive investigation in its evaluation.

Out of 16 intraoperatively proven cases 14 cases were diagnosed by USG -Doppler and 2 normal cases were misdiagnosed as invasive placenta. Thus, Sensitivity $-87.5 \%$, Specificity - 91.6\%, PPV - 87.5\%, NPV - 91.6\%

Out of 16 intraoperatively proven cases 15 cases were diagnosed correctly by MRI and 1 normal case was misdiagnosed as invasive placenta. Thus, Sensitivity was 93\%, Specificity- 95.8\%, PPV - 93\%\%, NPV - 95.8\%

\section{CONCLUSION}

MRI is more sensitive and specific than USG-Colour Doppler in diagnosing Invasive placenta. MRI has superior role compared to USG in evaluating Placenta percreta and invasion in cases of posteriorly located placenta previa. MRI has higher Positive and Negative Predictive value in diagnosing invasive placenta compared to USG Doppler. Most common finding in USG is presence of multiple intraplacental lacunae and loss of retro placental lucency.Most common finding in Doppler is turbulent flow in lacunae. Most common finding in MRI is presence of Intraplacental T2 Dark bands and heterogeneous signal intensity.

\section{REFERENCES}

1. Hashem LB, Salem DS, Hamed ST, Hussein AM. Role of MRI versus ultrasound in the assessment of placental abnormalities and diseases. The Egyptian Journal of Radiology and Nuclear Medicine. 2016;47(2):641-58.

2. Kumar I, Verma A, Ojha R, Shukla RC, Jain M, Srivastava A. Invasive placental disorders: a prospective US and MRI comparative analysis. Acta Radiologica. 2017;58(1):121-8.

3. Finberg HJ, Williams JW. Placenta accreta: prospective sonographic diagnosis in patients with placenta previa and prior cesarean section. Journal of ultrasound in medicine. 1992;11(7):333-43.

4. Twickler DM, Lucas MJ, Balis AB, Santos-Ramos R, 
Martin L, Malone S, Rogers B. Color flow mapping for myometrial invasion in women with a prior cesarean delivery. Journal of maternal-fetal medicine. 2000;9(6):330-5.

5. Maynard H, Zamudio S, Jauniaux E, Collins SL. The importance of bladder volume in the ultrasound diagnosis of placenta accreta spectrum disorders. International Journal of Gynecology \& Obstetrics. 2018;140(3):332-7.

6. Chou MM, Ho ES, Lee YH. Prenatal diagnosis of placenta previa accreta by transabdominal color Doppler ultrasound. Ultrasound in Obstetrics and Gynecology. 2000;15(1):28-35.

7. Chan BC,Lam HS, Yuen JH, Lam TP,Tso WK, Pun TC, Lee CP. Conservative management of placenta praevia with accreta. Hong Kong Med J. 2008;14(6):479-84.

8. Warshak CR, Eskander R, Hull AD, Scioscia AL, Mattrey RF, Benirschke K, Resnik R. Accuracy of ultrasonography and magnetic resonance imaging in the diagnosis of placenta accreta. Obstetrics \& Gynecology. 2006;108(3):573-81.

9. Dwyer BK, Belogolovkin V, Tran L, Rao A, Carroll I, Barth R, Chitkara U. Prenatal diagnosis of placenta accreta: sonography or magnetic resonance imaging. Journal of Ultrasound in Medicine. 2008;27(9):1275-81

10. Chen T, Xu XQ, Shi HB, Yang ZQ, Zhou X, Pan Y. Conventional MRI features for predicting the clinical outcome of patients with invasive placenta. Diagnostic and Interventional Radiology. 2017;23(3):173.

Source of Support: Nil; Conflict of Interest: None

Submitted: 27-09-2019; Accepted: 10-11-2019; Published online: 25-12-2019 